
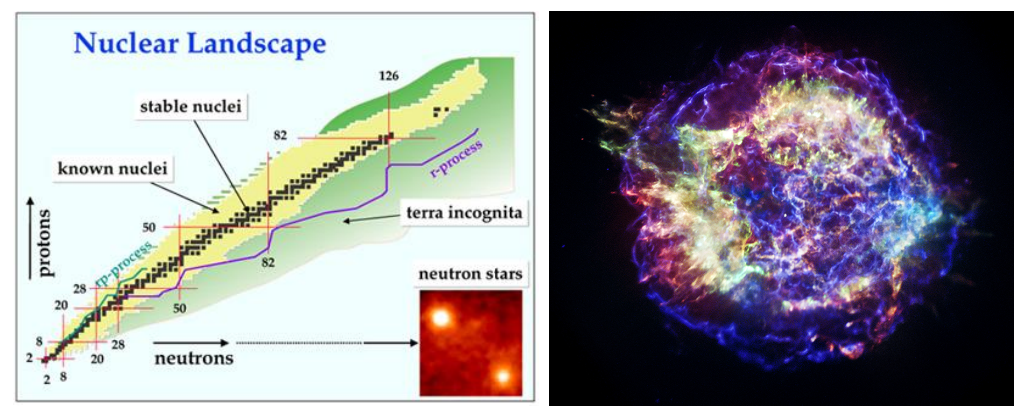

\title{
Relativistic density functional theory for finite nuclei and neutron stars ${ }^{T}$
}

\author{
J. Piekarewicz \\ Department OF PHYSICS \\ FLORIDA State University \\ TAllahassee, FL 32306
}

February 6, 2015

\begin{abstract}
The main goal of the present contribution is a pedagogical introduction to the fascinating world of neutron stars by relying on relativistic density functional theory. Density functional theory provides a powerful - and perhaps unique - framework for the calculation of both the properties of finite nuclei and neutron stars. Given the enormous densities that may be reached in the core of neutron stars, it is essential that such theoretical framework incorporates from the outset the basic principles of Lorentz covariance and special relativity. After a brief historical perspective, we present the necessary details required to compute the equation of state of dense, neutron-rich matter. As the equation of state is all that is needed to compute the structure of neutron stars, we discuss how nuclear physics - particularly certain kind of laboratory experiments - can provide significant constrains on the behavior of neutron-rich matter.
\end{abstract}

\footnotetext{
${ }^{1}$ Contributing chapter to the book "Relativistic Density Functional for Nuclear Structure"; World Scientific Publishing Company (Singapore); Editor Prof. Jie Meng.
} 


\section{INTRODUCTION}

The birth of a star is marked by the conversion of hydrogen into helium nuclei ( $\alpha$ particles) in their hot dense cores. This thermonuclear reaction is the main source of energy generation during the main stage of stellar evolution and provides the pressure support against gravitational collapse. Once the hydrogen in the stellar core is exhausted, thermonuclear fusion stops and the star contracts. As a result of the gravitational contraction, the temperature in the stellar core increases to about 100 million K allowing the heavier helium ashes to overcome their electrostatic repulsion and fuse into heavier elements. However, the absence of stable nuclei containing either five or eight nucleons hinders the production of heavy elements. Remarkably, the conditions of density and temperature in the stellar interior are such that a minute equilibrium concentration of ${ }^{8} \mathrm{Be}$ develops; the concentration of ${ }^{8} \mathrm{Be}$ relative to that of ${ }^{4} \mathrm{He}$ is about 1 parts per billion! Yet this minute concentration is sufficient for another $\alpha$-particle to be captured leading to the formation of a ${ }^{12} \mathrm{C}$ nucleus. The physics of the "triple-alpha" reaction, including the prediction of the resonant Hoyle state, is one of the most fascinating chapters in the story of stellar nucleosynthesis [1, 2]. Although in stars as our Sun the formation of heavier elements is hindered by the degeneracy pressure of the electrons, the conditions in the core of more massive stars are conducive to the formation of heavier elements, such as ${ }^{16} \mathrm{O},{ }^{24} \mathrm{Mg},{ }^{28} \mathrm{Si},{ }^{32} \mathrm{~S}$. However, abruptly and unavoidably, the fusion of light nuclei into ever increasing heavier elements terminates with the synthesis of the iron-group elements ( $\mathrm{Fe}, \mathrm{Co}$, and $\mathrm{Ni}$ ) that are characterized by having the largest binding energy per nucleon. That is, once iron-group elements are produced in the stellar interior, it is no longer feasible to generate energy by thermonuclear fusion. Indeed, if the iron core exceeds the "Chandrasekhar limit" of about 1.4 solar masses, neither thermonuclear fusion nor electron degeneracy pressure can prevent the rapid collapse of the stellar core. The collapse of the core, with the ensuing shock wave that disseminates the chemical elements crafted during the lifetime of the star, produces one of the most remarkable events in the Universe: a Supernova Explosion (see Fig.11). Besides creating an ejecta that contains some of the essential elements necessary for life, core-collapse supernovae leave behind exotic compact remnants in the form of either black holes or neutron stars. Neutron stars are the central theme of the present contribution.

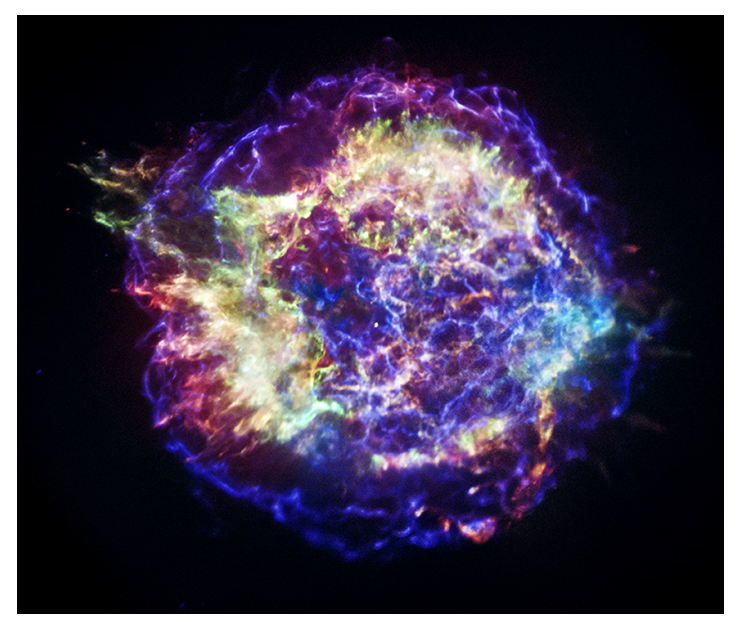

FIG. 1. Cassiopeia A is the remnant of a supernovae explosion that was witnessed in the northern sky about 300 years ago. The supernova remanent is located about 10,000 light years away from Earth and the small "dot" near the center of the image represents the dense neutron star. The image was created using NASA's Chandra x-ray observatory, an observatory named in honor of Subramanyan Chandrasekhar.

Indirectly and inadvertently, Subramanyan Chandrasekhar ("Chandra") may have been the discoverer of neutron stars. In a pioneering paper published in 1931, Chandra re-examined the role of electron degeneracy pressure in supporting a white-dwarf star against gravitational collapse, a fact that was already well known at the time. Chandra realized, however, that as the electrons become relativistic, the pressure support weakens and a white-dwarf star with a mass in excess of about 1.4 solar masses (the so-called "Chandrasekhar mass limit") will collapse under its own weight [3]. Chandra summarized eloquently this critical finding: For a star of small mass the white-dwarf stage is an initial step towards complete extinction. A star of large mass cannot pass into the white-dwarf stage and one is left speculating on other possibilities. One may ask why did Chandra never speculated that neutron stars may be among the "other possibilities". As luck will have it, the neutron was not yet discovered in 1931; it would take Chadwick another year to announce the discovery [4. However, soon after Chadwick's announcement, the term neutron star appears in writing for the first time in the 1933 proceedings of the the American Physical Society by Baade and 
Zwicky who wrote: With all reserve we advance the view that supernovae represent the transition from ordinary stars into "neutron stars", which in their final stages consist of extremely closed packed neutrons [5].

It appears, however, that speculations on the possible existence of both the neutron and neutron stars may have an earlier origin. In the case of the neutron, the story starts with the "boys of Via Panisperna" who included such luminaries as Enrico Fermi, Franco Rasetti, and Ettore Majorana, among others. During a brief stay at Caltech (in the 1928-29 period) working in Millikan's laboratory, Rasetti measured the ground-state angular momentum of ${ }^{14} \mathrm{~N}$ to be $J=1$. At the time, the widespread believe was that the nucleus of ${ }^{14} \mathrm{~N}$ must contain 14 protons (to account for its mass) and 7 electrons (to account for its charge). However, Majorana was the first one to realize that 21 spin- $1 / 2$ fermions can not account for the measured spin of ${ }^{14} \mathrm{~N}$, thereby postulating the existence of an electrically neutral spin-1/2 particle having the same mass as the proton [6. Moreover, the requirement for such a particle fitted correctly Majorana's interpretation of some experiments carried out in 1932 by Irène Joliot-Curie and Frédéric Joliot. Apparently Fermi pleaded with Majorana to write an article on the neutron, but Majorana did not find it worthy.
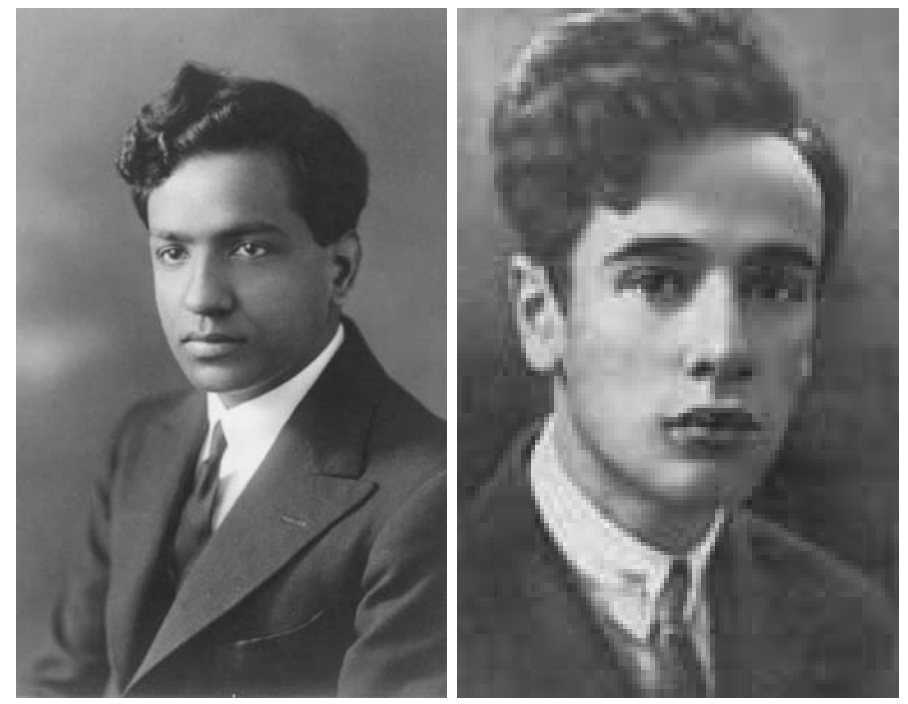

FIG. 2. Subrahmanyan Chandrasekhar (left) and Lev Landau (right) who wrote seminal papers on the theory of stars at the "ripe" age of 19 and 23, respectively.

Insofar as Landau's role on the history of neutron stars is concerned, the first recollection appears on a paper entitled "On the theory of stars" that Landau submitted for publication in early 1932 at the age of 23 [7]. In that paper Landau calculates - independently of Chandrasekhar - the maximum mass of a white dwarf star. Moreover, unlike Chandrasekhar, Landau did speculate on "other possibilities", namely, the existence of dense stars that look like giant atomic nuclei. For an in depth and fascinating tale on Landau's role on the possible existence of neutron stars see Ref. [8]. Note that in Fig. 2 we display pictures of both Chandra and Landau as very young men.

Perhaps the last great theoretical landmark of that time involves the 1939 work by Oppenheimer and Volkoff on the structure of neutron stars [9]. By then, Einstein's general theory of relativity was firmly established as was Tolman's framework to compute solutions appropriate to spherical systems in hydrostatic equilibrium [10]. In what it is now referred to as the Tolman-Volkoff-Oppenheimer (TOV) equations - effectively the generalization of Newtonian gravity to the domain of general relativity - Oppenheimer and Volkoff concluded that a neutron star supported exclusively by the pressure from its degenerate neutrons will collapse into (what we now know as) a black hole for masses in excess of about 0.7 solar masses. This critical finding, together with our present knowledge of neutron-star masses, has made nuclear physics and astrophysics intimately intertwined.

Although firmly established theoretically, it would take almost three decades for the discovery of neutron stars. This momentous discovery started with a young graduate student by the name of Jocelyn Bell (see Fig. 3) — now Dame Jocelyn Bell Burnell - who detected a "bit of scruff" in the data arriving into her radio telescope, a telescope originally designed to study distant quasars. The arriving signal was "pulsing" with such an enormous regularity, indeed once every 1.337302088331 seconds that both Bell and her research advisor, Anthony Hewish, were so mesmerized by the observation that they were convinced that the signal was a beacon from an extraterrestrial civilization. Initially dubbed as "Little Green Man 1" the source, now known as radio pulsar "PSR B1919+21", was shortly identified as a rapidly rotating neutron star 11]. Although Hewish was recognized with the Physics Nobel Prize in 1974 for "his decisive role in the discovery of pulsars", Jocelyn Bell did not share the award. The exclusion of Jocelyn Bell as co-recipient of the Nobel Prize was both controversial and roundly condemned by the astrophysics community. Still, 


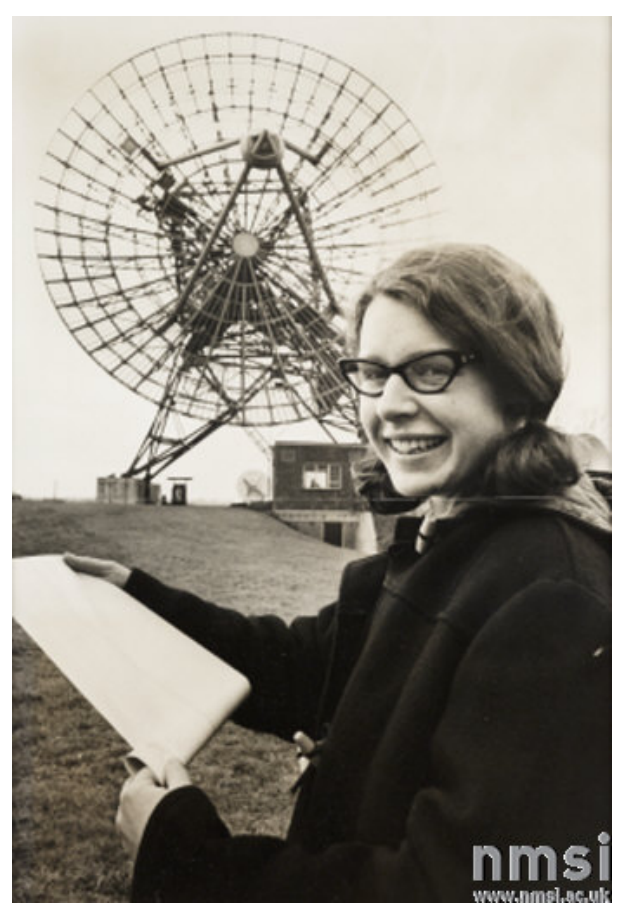

FIG. 3. Jocelyn Bell as a young graduate student with her radio telescope designed to search for quasars. Instead, and according to Dr. Iosif Shklovsky, she made the greatest astronomical discovery of the twentieth century.

Bell has always displayed enormous grace and humility in the face of this controversy. Indeed, she has stated: I believe it would demean Nobel Prizes if they were awarded to research students, except in very exceptional cases, and I do not believe this is one of them. It appears that Dr. Iosif Shklovsky, as well as many others, did not share her views. Dr. Shklovsky — recipient of the 1972 Bruce Medal for outstanding lifetime contributions to astronomy—told Jocelyn Bell: Miss Bell, you have made the greatest astronomical discovery of the twentieth century.

TABLE I. Approximate characteristics of a "canonical" neutron star, such as the 960 year old Crab pulsar.

\begin{tabular}{ll}
\hline \hline Name: PSR B0531+21 & Constellation: Taurus \\
Distance: $2.2 \mathrm{kpc}$ & Age: 960 years \\
Mass: $1.4 M_{\odot}$ & Radius: $10 \mathrm{~km}$ \\
Density: $10^{15} \mathrm{~g} / \mathrm{cm}^{3}$ & Pressure: $10^{29} \mathrm{~atm}$ \\
Surface Temperature: $10^{6} \mathrm{~K}$ & Escape velocity: $0.6 \mathrm{c}$ \\
Period: $33 \mathrm{~ms}$ & Magnetic Field: $10^{12} \mathrm{G}$ \\
\hline \hline
\end{tabular}

The finding by Oppenheimer and Volkoff of a maximum neutron star mass of about 0.7 solar masses 9 will eventually place nuclear physics at the forefront of neutron-star structure. This is because the masses of various neutron stars have been determined very accurately and they exceed, often by a significant margin, the $0.7 M_{\odot}$ limit (see Ref. [12] and references contained therein). Indeed, the present "record" stands at $2 M_{\odot}$ [13, 14]. Given that the OppenheimerVolkoff limit was obtained by assuming that the support against gravitational collapse is provided by a degenerate gas of neutrons, the large deficit must then be supplied by nuclear interactions. Thus, neutron stars are enormously valuable in constraining the largely undetermined equation of state of neutron-rich matter at high densities. However, the reach of neutron stars is not limited to the nuclear physics domain. Indeed, neutron stars are unique laboratories for the study of matter under extreme conditions of density and isospin asymmetry. In particular, their extreme compactness has been used to test the basic tenets of general relativity [15. Moreover, by spanning many orders of magnitude in density, neutron stars display exotic phases that cannot be realized under normal laboratory conditions. Yet, some of these phases have direct counterparts in both atomic physics and condensed-matter physics. Finally, their very dense stellar cores may harbor novel states of matter, such as color superconductors, that are a direct prediction of Quantum Chromodynamics [16. To appreciate some of the unique properties of neutron stars, such as their density, pressure, spin period, and magnetic fields, we have listed some of these properties in TableI for the case of the well-known crab pulsar. 
We have organized this chapter on neutron stars as follows. After this Introduction, we present the formalism that will be used to compute some structural properties of neutron stars, such as their mass-vs-radius relation. Once the formalism is in place, we embark on a journey of a neutron star that involves a detailed discussion of the outer crust, the inner crust, and the outer core. For all these three cases we present results that highlight those stellar observables that are particularly sensitive to the choice of the nuclear density functional. We finish with a summary of our results and an outlook for the future.

\section{FORMALISM}

The ultimate goal of a properly constructed nuclear energy density functional is to provide a unified description of a wide variety of physical phenomena ranging from the properties of finite nuclei to the structure and dynamics of neutron stars. This ambitious goal involves physical systems that differ in mass and size by about 55 and 18 orders of magnitude, respectively. Moreover, given that extrapolations into regions that are inaccessible in laboratory experiments are unavoidable, the predictions of such microscopic theory should always be accompanied by wellquantified theoretical uncertainties [17, 18.

Historically, relativistic models of nuclear structure were limited to renormalizable field theories [19, 20]. The appeal of renormalizability was evident: with only a handful of model parameters calibrated to well-known physical observables one could then extrapolate to unknown physical regions without the need for introducing additional parameters. However, the modern viewpoint suggests that any relativistic model, although often inspired in Quantum Chromodynamics (QCD), should be treated as an effective field theory (EFT) where the demand for renormalizability is no longer required. An effective field theory is designed to describe low-energy physics without any attempt to account for its detailed short-distance behavior [21]. Although in principle the empirical parameters of the EFT may be calculable from QCD, in practice this becomes enormously challenging in the non-perturbative regime of relevance to nuclear systems. Hence, the parameters of the model are directly calibrated (i.e., fitted) to physical observables. By doing so, the short-distance structure of the theory as well as many other complicated many-body effects get implicitly encoded in the parameters of the model. In this regard, density functional theory (DFT), a powerful and highly successful theoretical framework pioneered by Kohn and collaborators [22 24, provides a unified approach for the construction of an EFT that may be used to compute phenomena ranging over many distance scales.

\section{A. Relativistic Density Functional Theory}

In the framework of the relativistic density functional theory, the effective degrees of freedom include nucleons (protons and neutrons), three "mesons", and the photon. The interactions among the particles can be described as a generalization of the original Lagrangian density of Serot and Walecka 19, 25, 28. That is,

$$
\begin{aligned}
\mathscr{L}_{\text {int }} & =\bar{\psi}\left[g_{\mathrm{s}} \phi-\left(g_{\mathrm{v}} V_{\mu}+\frac{g_{\rho}}{2} \boldsymbol{\tau} \cdot \mathbf{b}_{\mu}+\frac{e}{2}\left(1+\tau_{3}\right) A_{\mu}\right) \gamma^{\mu}\right] \psi \\
& -\frac{\kappa}{3 !}\left(g_{\mathrm{s}} \phi\right)^{3}-\frac{\lambda}{4 !}\left(g_{\mathrm{s}} \phi\right)^{4}+\frac{\zeta}{4 !} g_{\mathrm{v}}^{4}\left(V_{\mu} V^{\mu}\right)^{2} \\
& +\Lambda_{\mathrm{v}}\left(g_{\rho}^{2} \mathbf{b}_{\mu} \cdot \mathbf{b}^{\mu}\right)\left(g_{\mathrm{v}}^{2} V_{\nu} V^{\nu}\right),
\end{aligned}
$$

where $\psi$ is the isodoublet nucleon field, $A_{\mu}$ is the photon field, and $\phi, V_{\mu}$, and $\mathbf{b}_{\mu}$ represent the isoscalar-scalar $\sigma$-, isoscalar-vector $\omega$-, and isovector-vector $\rho$-meson field, respectively. We note that the pion is not explicitly included in the Lagrangian density as it does not contribute to the nuclear dynamics at the mean field level. The Lagrangian density incorporates the conventional Yukawa terms between the nucleon and the various mesons and the photon. However, in order to improve the quality of the model it is critical to supplement the dynamics with nonlinear interaction terms between the various mesons. In the spirit of an effective field theory, one should incorporate all possible meson interactions that are allowed by symmetry considerations to a given order in a power-counting scheme. Moreover, once the dimensionful meson fields have been properly scaled using strong-interaction mass scales, the remaining dimensionless coefficients of the effective Lagrangian should all be "natural", namely, neither too small nor too large 29 33. However, given the limited experimental database of nuclear observables, certain empirical coefficients - or linear combinations of them-may remain poorly determined even after the optimization procedure. This results in "unnatural" coefficients that deviate significantly from unity. Therefore, in an effort to avoid this problem, only those nonlinear meson interactions with a clear physical interpretation are retained. For instance, for the Lagrangian density depicted in Eq. (1), we have only kept the four non-linear meson interactions that are denoted by the coefficients: $\kappa, \lambda, \zeta$, and $\Lambda_{\mathrm{v}}$. Two of the isoscalar parameters, $\kappa$ and $\lambda$, were introduced by 
Boguta and Bodmer 34 to soften the equation of state of symmetric nuclear matter, primarily the incompressibility coefficient [19, 25], in an effort to make the theory consistent with measurements of giant monopole resonances in finite nuclei. In turn, $\zeta$ may be used to efficiently tune the maximum neutron star mass without sacrificing the agreement with other well reproduced observables [26. Finally, $\Lambda_{\mathrm{v}}$ is highly sensitive to the density dependence of symmetry energy - and in particular to its slope at saturation density - which has important implications in the structure and dynamics of neutron stars $28,35,37$.

With the Lagrangian density given in Eq. (1), one can derive the equations of motion for each of the constituent particles in the mean-field approximation [38. In particular, the nucleons satisfy a Dirac equation in the presence of mean-field potentials of Lorentz scalar and vector character. In turn, the various meson fields satisfy both nonlinear and inhomogeneous Klein-Gordon equations with the various nuclear densities acting as source terms. Given that the nuclear densities act as sources for the meson fields and, in turn, the meson fields determine the mean-field potentials for the nucleons, the set of equations must be solved self-consistently. Once solved, these equations determine the ground-state properties of the nucleus of interest - such as its total binding energy, the single-nucleon energies and Dirac orbitals, the distribution of meson fields, and the various density profiles.

\section{B. Nuclear Matter Equation of State}

The solution of the mean-field equations is simplified considerably in the case of infinite nuclear matter, which is assumed to be spatially uniform. Although the solution may be found at finite temperature, our main goal is to solve the mean-field equations at zero temperature because of their relevance to the structure and dynamics of neutron stars. Indeed, if the validity of Einstein's theory of General Relativity is assumed, then the equation of state of asymmetric nuclear matter represents the sole ingredient required to compute the properties of neutron stars (see next section).

In the simplified case of infinite nuclear matter, the meson fields are uniform (i.e., constant throughout space) and the nucleon orbitals are plane-wave Dirac spinors with medium-modified effective masses and energies that must be determined self-consistently. By constructing the energy-momentum tensor in the mean-field approximation [25], one obtains the equation of state of asymmetric nuclear matter, namely, the energy density and pressure of the system as a function of both the conserved baryon density $\rho=\rho_{n}+\rho_{p}$ and the neutron-proton asymmetry $\alpha \equiv\left(\rho_{n}-\rho_{p}\right) /\left(\rho_{n}+\rho_{p}\right)$. A particularly insightful view of the EOS is obtained by expanding the energy per nucleon in powers of the neutronproton asymmetry. That is,

$$
\frac{E}{A}(\rho, \alpha)-M \equiv \mathcal{E}(\rho, \alpha)=\mathcal{E}_{\mathrm{SNM}}(\rho)+\alpha^{2} \mathcal{S}(\rho)+\mathcal{O}\left(\alpha^{4}\right) .
$$

Here $\mathcal{E}_{\mathrm{SNM}}(\rho)=\mathcal{E}(\rho, \alpha \equiv 0)$ is the energy per nucleon of symmetric nuclear matter (SNM) and the symmetry energy $\mathcal{S}(\rho)$ represents the first-order correction to the symmetric limit. Note that no odd powers of $\alpha$ appear as the nuclear force is assumed to be isospin symmetric and long-range electromagnetic effects have been "turned off". Also note that to a very good approximation the symmetry energy represents the energy cost required to convert symmetric nuclear matter into pure neutron matter (PNM). That is,

$$
\mathcal{S}(\rho) \approx \mathcal{E}(\rho, \alpha=1)-\mathcal{E}(\rho, \alpha=0) .
$$

Such a separation is useful because symmetric nuclear matter is sensitive to the isoscalar sector of the density functional which is well constrained by the properties of stable nuclei. In contrast, the symmetry energy probes the isovector sector of the density functional which at present is poorly constrained. However, this problem will be mitigated with the commissioning of radioactive beam facilities throughout the world.

Besides the separation of the EOS into symmetric and asymmetric components, it is also useful to characterize the behavior of the equation of state in terms of a few bulk parameters. To do so one performs a Taylor series expansion around nuclear matter saturation density $\rho_{0}$. That is [39],

$$
\begin{aligned}
& \mathcal{E}_{\mathrm{SNM}}(\rho)=\varepsilon_{0}+\frac{1}{2} K_{0} x^{2}+\ldots \\
& \mathcal{S}(\rho)=J+L x+\frac{1}{2} K_{\mathrm{sym}} x^{2}+\ldots
\end{aligned}
$$

where $x=\left(\rho-\rho_{0}\right) / 3 \rho_{0}$ is a dimensionless parameter that quantifies the deviations of the density from its value at saturation. Here $\varepsilon_{0}$ and $K_{0}$ represent the energy per nucleon and the incompressibility coefficient of SNM; $J$ and $K_{\text {sym }}$ are the corresponding quantities for the symmetry energy. However, unlike symmetric nuclear matter whose 
pressure vanishes at $\rho_{0}$, the slope of the symmetry energy $L$ does not vanish at saturation density. Indeed, assuming the validity of Eq. (3), $L$ is directly proportional to the pressure of PNM $\left(P_{0}\right)$ at saturation density, namely,

$$
P_{0} \approx \frac{1}{3} \rho_{0} L
$$

In computing various neutron-star observables in the next few sections, we will rely on several nuclear density functionals that while successful in reproducing a myriad of laboratory observables, predict significant differences in the properties of neutron stars.

\section{Tolman-Oppenheimer-Volkoff Equations}

With masses comparable to that of our Sun but with radii that are almost five orders of magnitude smaller (i.e., of the order of $10 \mathrm{~km}$ ) neutron stars are highly compact objects that must be described using Einstein's theory of General Relativity. The generalization of Newtonian gravity to the realm of general relativity is expressed in the Tolman-Oppenheimer-Volkoff (TOV) equations, which are usually presented as a coupled set of first-order differential equations of the following form:

$$
\begin{aligned}
& \frac{d P}{d r}=-G \frac{\mathcal{E}(r) M(r)}{r^{2}}\left[1+\frac{P(r)}{\mathcal{E}(r)}\right]\left[1+\frac{4 \pi r^{3} P(r)}{M(r)}\right]\left[1-\frac{2 G M(r)}{r}\right]^{-1} \\
& \frac{d M}{d r}=4 \pi r^{2} \mathcal{E}(r),
\end{aligned}
$$

where $G$ is Newton's gravitational constant and $P(r), \mathcal{E}(r)$, and $M(r)$ represent the pressure, energy density, and enclosed-mass profiles of the star, respectively. Note that the three terms enclosed in square brackets in Eq. (6) are of general-relativistic origin. As already alluded earlier and particularly interesting, the only input that neutron stars are sensitive to is the equation of state of neutron-rich matter. This fact alone creates a unique synergy between nuclear physics and astrophysics. In essence, by specifying the central pressure and enclosed mass, i.e., $P_{c}=P(r=0)$ and $M(r=0)=0$ - together with a suitable EOS - the TOV equations may be solved using a standard numerical algorithm, such as the Runge-Kutta method.

\section{ANATOMY OF A NEUTRON STAR}

In the next few sections we embark on a journey through a neutron star. According to Baade and Zwicky, the most common perception of a neutron star is that of a uniform assembly of extremely closed packed neutrons [5]. We will now show, however, how the reality is far different and much more interesting. In particular, as we journey through the neutron star we will discover a myriad of exotic states of matter and will discuss the critical role that laboratory experiments can play in elucidating their fascinating nature. Because of their enormous relevance to nuclear physics, we focus our attention on three components of the neutron star: (a) the outer crust, (b) the inner crust, and (c) the outer core. For two recent reviews on the exotic nature of the stellar crust see [40, 41] and references therein. The three regions are clearly highlighted in Fig. 4, which includes two physically accurate renditions of a neutron star. In particular, the question mark at the center of the left-hand illustration denotes the possibility that the inner stellar core harbors exotic states of matter, such as hyperons, meson condensates, and strange quark matter. Although this idea is enormously provocative, at present there is simply not enough experimental information to properly constrain the dynamics of the inner core. Thus, the possible existence of such exotic states of matter will be ignored hereafter. Although the stellar atmosphere and the envelope will also be ignored, we briefly discuss now some of its most relevant features.

Because of the enormous gravitational fields around a neutron star, the atmosphere is believed to be about a mere $10 \mathrm{~cm}$ thick. However, the atmosphere shapes the thermal radiation from the photosphere which is customarily assumed to be that of a black body. Hence, detailed knowledge of the atmosphere is critical for the reliable extraction of, for example, stellar radii. In turn, the $100 \mathrm{~m}$ envelope acts as a blanket that modulates the huge temperature gradient between the core and the crust. For more information about the role of the stellar atmosphere and envelope see Ref. [42] and references contained therein. 

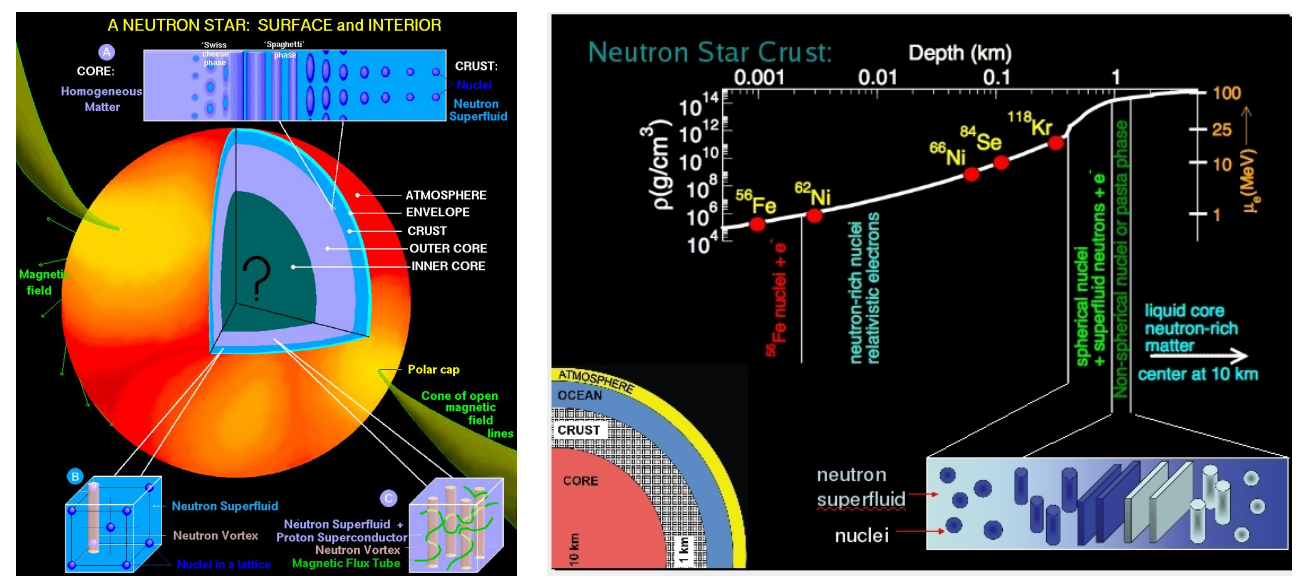

FIG. 4. Two physically accurate renditions of the structure, composition, and possible phases of a neutron star. Courtesy of Dany Page (left panel) and Sanjay Reddy (right panel).

\section{A. The Outer Crust}

The outer crust of a neutron star comprises a region that spans almost 7 orders of magnitude in baryon density; from about $10^{4} \mathrm{~g} / \mathrm{cm}^{3}$ to $4 \times 10^{11} \mathrm{~g} / \mathrm{cm}^{3}$ [3]. Recall that nuclear-matter saturation density is $\rho_{0} \simeq 2.4 \times 10^{14} \mathrm{~g} / \mathrm{cm}^{3}$, which corresponds to a baryon density of $n_{0} \simeq 0.15 \mathrm{fm}^{-3}$. Thus, these densities are significantly lower that those encountered in the interior of the atomic nucleus. However, at these densities the electrons - which represent a critical component of the star in order to maintain the overall charge neutrality of the system-have been pressure ionized. Thus, they can be accurately described by a relativistic free Fermi gas. Moreover, given that at these densities the average inter-nucleon distance is significantly larger than the range of the strong nuclear force, the uniform ground state becomes unstable against cluster formation. That is, under these conditions of density it is energetically favorably for translational invariance to be broken and for the individual nucleons to cluster into "normal" nuclei. Thus, the outer stellar crust consists of isolated nuclei embedded in a uniform electron gas. Moreover, because the short-range nuclear force saturates within the individual clusters, nuclei interact exclusively via the long-range Coulomb interaction. This promotes the formation of a Coulomb crystal of nuclei arranged in a body-centered-cubic (bcc) lattice that itself is embedded in a neutralized uniform electron gas [43. In the particular case of the top layers of the crust where the density is at its lowest, the energetically preferred nucleus is ${ }^{56} \mathrm{Fe}$; see the right-hand panel of Fig. 44. Recall that ${ }^{56} \mathrm{Fe}$ is the nucleus with the lowest mass per nucleon.

However, as one moves inward towards the center of the star, the density increases, and so does the electronic contribution to the total energy. Thus, it becomes energetically advantegeous to remove a fraction of the electrons (through electron capture) albeit at the expense of an increase in the neutron-proton asymmetry. In this manner, the energetically most favorable nucleus emerges from a competition between the electronic contribution, which favors a small electron (and proton) fraction, and the nuclear symmetry energy which, in turn, favors symmetric nuclei. Hence, the nuclear contribution to the composition of the outer crust appears in the form of a nuclear mass table that is generated from a combination of experimental data and theoretical predictions. Indeed, in full thermodynamic equilibrium, one determines the crustal composition by minimizing the chemical potential of the system $(\mu)$ at zero temperature and fixed pressure. That is [4,

$$
\mu(A, Z ; P)=\frac{M(N, Z)}{A}+\frac{Z}{A} \mu_{e}-\frac{4}{3} C_{\ell} \frac{Z^{2}}{A^{4 / 3}} p_{\mathrm{F}} .
$$

The total chemical potential consists of nuclear, electronic, and lattice contributions. As already mentioned, computing the nuclear contribution requires of "only" a reliable nuclear mass table. Moreover, given that at the relevant densities the electrons can be accurately modeled by a Fermi-gas distribution, its contribution to the chemical potential is simply given by

$$
\mu_{e}=\sqrt{\left(p_{\mathrm{F}}^{e}\right)^{2}+m_{e}^{2}}=\sqrt{\left(y p_{\mathrm{F}}\right)^{2}+m_{e}^{2}},
$$

where $y=Z / A$ is the electron fraction and the Fermi momentum $p_{\mathrm{F}}$ is related to the baryon density $n$ by

$$
p_{\mathrm{F}}=\left(3 \pi^{2} n\right)^{1 / 3} .
$$


Finally, the last term in Eq. (8) represents the complicated lattice contribution. This contribution is complex because it involves the long-range nature of the Coulomb interaction. Nevertheless, the overall charge neutrality of the system ensures that the contribution is finite [45, 46. In the particular case of the energetically preferred bcc lattice, one obtains 43 ]

$$
\varepsilon_{\ell}(A, Z ; n)=-C_{\ell} \frac{Z^{2}}{A^{4 / 3}} p_{\mathrm{F}}
$$

where $C_{\ell}=3.40665 \times 10^{-3}$ is a dimensionless constant [4]. Note that although the expression for the chemical potential is written in terms of the density rather than the pressure, chemical equilibrium demands that the minimization of $\mu(A, Z ; P)$ be carried out at a fixed pressure rather than at a fixed density. Thus, one needs an equation of state to properly relate them. However, given that for the outer crust the pressure is dominated by the degenerate electrons (with only a small lattice contribution) to a very good approximation the relevant equation of state is that of a relativistic Fermi gas of electrons.
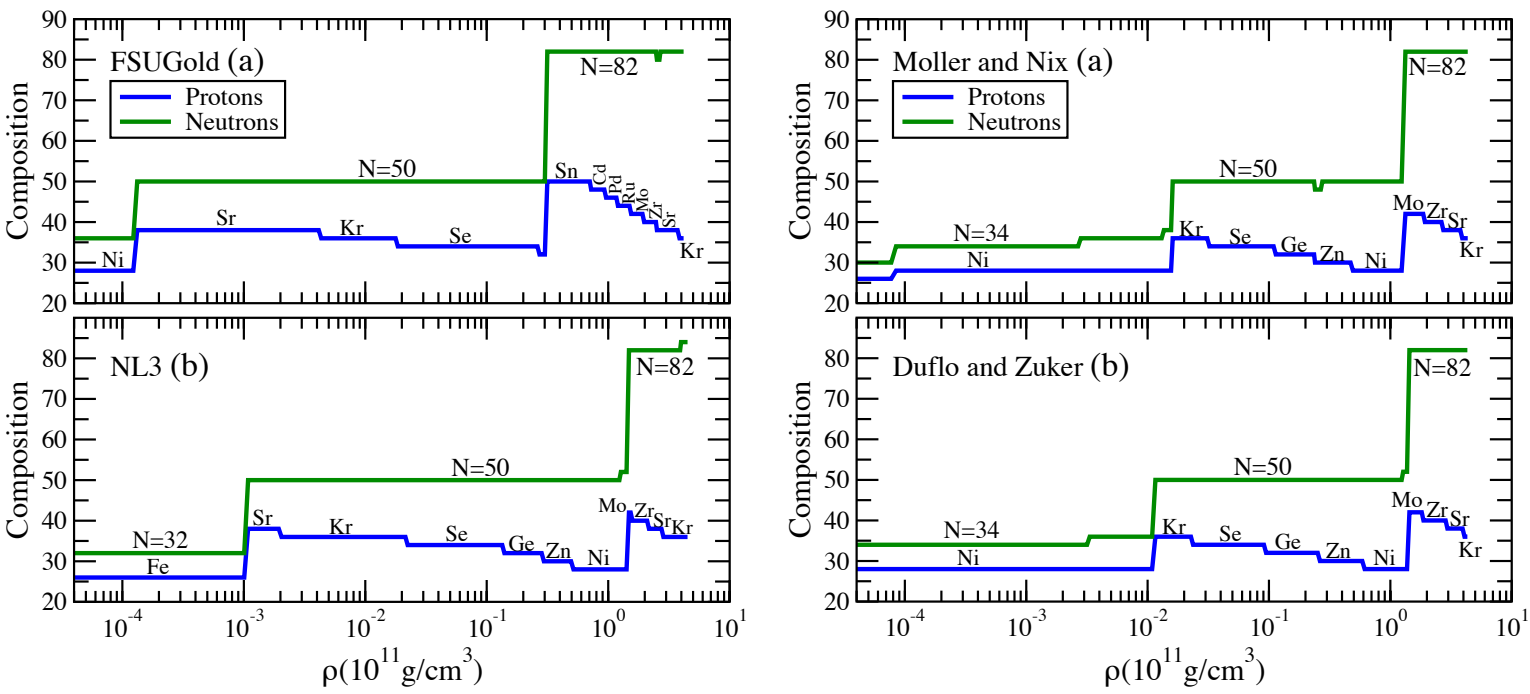

FIG. 5. Composition of the outer crust as predicted by two accurately-calibrated relativistic mean field models (left) and by two microscopic-macroscopic models (right). Protons are depicted with the blue (lower) line whereas neutrons with the green (upper) line.

To assess the sensitivity of the crustal composition to the mass model we display in Fig.5 predictions using two accurately calibrated relativistic EDFs, i.e., FSUGold [4] and NL3 [48, 49] as well as two highly successful microscopicmacroscopic (mic-mac) models; one due to Möller and collaborators [50, 51, and the other one due to Duflo and Zuker 52 54. As already anticipated, the top (i.e., most dilute) layers of the outer crust consist of a crystalline lattice of ${ }^{56} \mathrm{Fe}$ nuclei embedded in a uniform electron gas; for a more aesthetic view see the right-hand panel of Fig.4. However, as the density increases - and with it the electron chemical potential - it becomes energetically favorable to replace ${ }^{56} \mathrm{Fe}$ with a slightly more neutron-rich nucleus. That is, at slightly higher densities ${ }^{56} \mathrm{Fe}$ ceases to be the most stable nucleus. Rather, the slightly more isospin asymmetric (even-even) Nickel isotopes become energetically favored. As the density continues to increase further, the system must decide whether to reduce $Z$ at neutron number $N \approx 34$ or to increase both $N$ and $Z$ by jumping to the next magic shell at $N=50$. Although it is seen that all four models favor an eventual jump to the $N=50$ shell, the predictions for the density at which the jump occurs is highly model dependent. Indeed, whether FSUGold predicts the transition at a density of about $10^{7} \mathrm{~g} / \mathrm{cm}^{3}$, both mic-mac models suggest a density that is almost two orders of magnitude larger. Our results also indicate that in all cases the proton fraction decreases systematically with increasing density in an effort to reduce the electronic contribution to the chemical potential. Yet, at one point reducing the electron fraction even further becomes too expensive for the symmetry energy to sustain and the system jumps to the next plateau at magic number $N=82$. We want to underscore that both the transition density as well as the crustal composition depend sensitively on the symmetry energy at sub-saturation density.

In the particular case of the two microscopic models (FSUGold and NL3) the symmetry energy may be computed at all densities, so a study of the trends displayed in Fig.5 are illuminating. At a density of relevance to finite nuclei, which consists of an average between the nuclear interior and the nuclear surface, the symmetry energy is known to be larger for FSUGold than for NL3. This implies that a neutron-proton mismatch at those densities is more 
costly for FSUGold than for NL3 38. Thus, the tolerance to a larger neutron-proton asymmetry is responsible for delaying the transition to the next higher plateau for NL3 relative to FSUGold, a fact that is clearly evident in the figure. By the same token, NL3 predicts a more exotic crustal composition than FSUGold. Indeed, whereas FSUGold suggests the formation of ${ }^{132} \mathrm{Sn}$ at the transition to the $N=82$ shell, NL3 predicts the formation of the significantly more neutron-rich isotope ${ }^{124}$ Mo. Note that as the density continues to increase even further, the neutron-proton asymmetry will become so large that the neutron drip line will be reached. For all models, the drip line is predicted to occur at a density of about $4 \times 10^{11} \mathrm{~g} / \mathrm{cm}^{3}$ and with the formation of the highly exotic ${ }^{118} \mathrm{Kr}$ isotope.

We close this section with a brief comment on the assumptions and extrapolations required to predict the composition of the outer crust. As just described, there are three main regions in the nuclear chart that are of direct relevance to the outer crust: (a) The Fe-Ni region, (b) the $N=50$ isotones from $\mathrm{Ni}(Z=28)$ to $\mathrm{Sr}(Z=38)$, and (c) the $N=82$-isotone region from $\operatorname{Kr}(Z=36)$ to $\operatorname{Sn}(Z=50)$. In regards to (a), all the masses in this region have been measured with great precision [55. In the case of (b), precise mass values exist, but only for the cases of ${ }^{88} \mathrm{Sr}$, ${ }^{86} \mathrm{Kr}$, ${ }^{84} \mathrm{Se},{ }^{82} \mathrm{Ge}$, and ${ }^{80} \mathrm{Zn}$, but not for either ${ }^{78} \mathrm{Ni}$ or ${ }^{82} \mathrm{Zn}[55$, [56. However, in a pioneering Penning trap experiment with the ISOLTRAP setup at the ISOLDE-CERN facility, the mass of ${ }^{82} \mathrm{Zn}$ has been recently determined [57. This new mass determination has ruled out the presence of ${ }^{82} \mathrm{Zn}$ in the outer crust and provides the most stringent constraint to date on its composition profile. Finally, the $N=82$-isotone region remains largely unexplored and it is likely to remain so even after the construction of a new generation of rare isotope facilities. Thus, the only hope to elucidate the composition of the bottom layers of the outer crust is through theoretical modeling. In this regard, measuring a large number of as yet unknown masses of exotic nuclei - even if of no direct relevance to the composition of the outer crust - will still be instrumental in guiding the calibration of future nuclear density functionals.

\section{B. The Inner Crust}

The inner crust of a neutron star comprises the region from neutron-drip density up to the density at which uniformity in the system is restored; about one third to one half of normal nuclear density. However, the transition from the highly ordered Coulomb crystal to the uniform liquid is both interesting and complex. This is because distance scales that are well separated in both the crystalline phase, where the long-range Coulomb interaction dominates, and in the uniform phase, where the short-range strong interaction dominates, become comparable in the inner stellar crust. This unique situation involving competing distance scales gives rise to Coulomb frustration. Frustration, a universal phenomenon characterized by the existence of a very large number of low-energy configurations, emerges from the impossibility to simultaneously minimize all elementary interactions in the system. Ultimately, the competition between the short-range nuclear interaction and the long-range Coulomb repulsion results in the formation of complex topological structures collectively referred to as nuclear pasta. Given that these complex structures are very close in energy, it has been speculated that the transition from the highly ordered crystal to the uniform phase must proceed through a series of changes in the dimensionality and topology of these structures [58, 59. Moreover, due to the preponderance of low-energy states, frustrated systems display an interesting and unique low-energy dynamics. We note that a seemingly unrelated condensed-matter problem, namely, the strongly-correlated electron gas, also displays Coulomb frustration. In the case of the electron gas, one aims to characterize the transition from the low-density Wigner crystal, where the long-range Coulomb potential dominates, to the uniform Fermi liquid, where the kinetic energy dominates 60. It has been shown that such a transition must be mediated by the emergence of "microemulsions", namely, exotic pasta-like structures with interesting topologies. Indeed, it has been proven that in two-spatial dimensions a direct first-order phase transition is forbidden in the presence of long-range (e.g., Coulomb) forces 61 .

To illustrate the complexity of the pasta phase we display in Fig.6 two snapshots obtained from Monte-Carlo and Molecular-Dynamics simulations of a nuclear system at densities of relevance to the inner stellar crust [37, 62. The left-hand panel displays how at a density of about one sixth of normal nuclear density and a proton fraction of $Z / A=0.2$, the system organizes itself into neutron-rich clusters (i.e., "nuclei") of complex topologies that are surrounded by a dilute vapor of likely superfluid neutrons. In turn, the right-hand panel displays the complex topology of a proton iso-surface (i.e., a surface of constant proton density) for a system of 100,000 nucleons. Such complex pasta structures may have a significant impact on various transport properties, such as neutrino propagation and electron conductivity. We should underscore that the emergence of such complex structures is a true dynamical effect associated with Coulomb frustration, as no a-priori shapes (such as spheres, rods, slabs, etc.) are ever assumed.

A great advantage of molecular-dynamics (MD) simulations is that many-body correlations are properly accounted within the formalism [37, 62 66. However, given their classical nature, MD simulations fail to capture any quantummechanical detail that the system may be sensitive to; for example, the superfluid nature of the dilute neutron vapor. In contrast, mean-field approximations incorporate quantum-mechanical effects (at least on average) but fail 

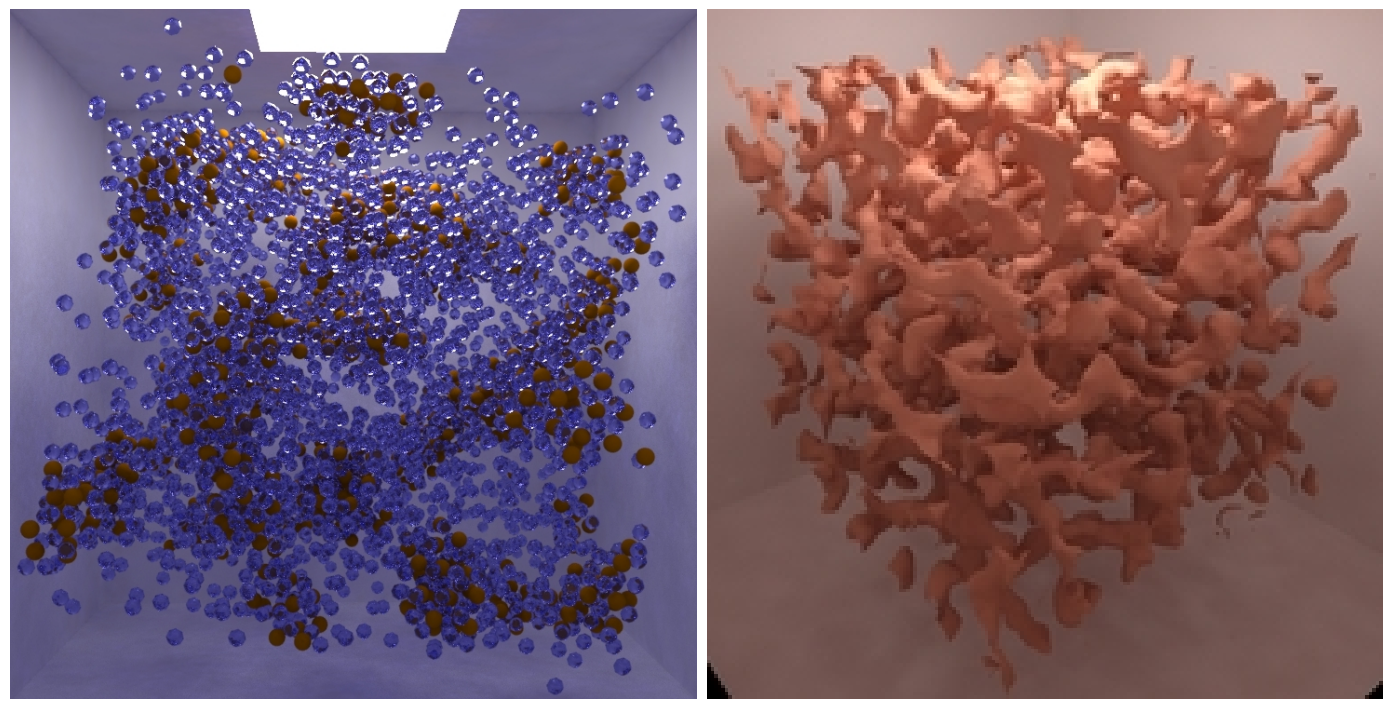

FIG. 6. (Left panel) A snapshot of a Monte Carlo simulation for one configuration of 4,000 nucleons at a baryon density of

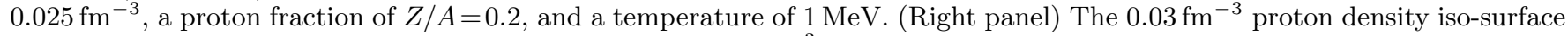
for one configuration of 100,000 nucleons at a density of $0.05 \mathrm{fm}^{-3}$, a proton fraction of $Z / A=0.2$, and a temperature of $1 \mathrm{MeV}$. Note that the simulation volume in this latter case is fairly large, i.e., $V=(126 \mathrm{fm})^{3}$.

to describe the important and complicated clustering correlations 67-71. Nevertheless, because the robustness of Coulomb frustration, both set of theoretical approaches seem to reach similar conclusions. For example, for relatively large proton fractions in the 0.3-0.5 range (such as in core-collapse supernovae) there appears to be general agreement that the transition from the ordered Coulomb crystal to the uniform phase must proceed via intermediate pasta phases. What is unclear, however, is whether such exotic pasta shapes can develop in the proton-poor environment characteristic of the inner stellar crust [72]. Note that mean-field models that impose $\beta$-equilibrium predict proton fractions at densities of relevance to the inner crust of only a few percent $67,69,71$.

Another enormous challenge associated with the purported pasta phases is the identification of a set of astrophysical observables that are sensitive to their formation. Colloquially, we refer to this challenge as "how to smell the pasta?" A possible manifestation of the nuclear pasta on the dynamics of neutron stars has been suggested recently by Pons, Viganò, and Rea [73. These authors have identified a special class of rotation-powered pulsars-the isolated x-ray pulsars - that appear to display spin periods shorter than about 12 seconds. Although enormously stable, rotationpowered pulsars are known to slow down, albeit very slowly, due to the emission of magnetic dipole radiation. The lack of isolated x-ray pulsars with spin periods longer than 12 seconds seems to suggest magnetic field decay due to the existence of a highly resistive layer in the inner crust; such a layer has been speculated to be the exotic nuclear pasta phase [73. Very recently, Horowitz and collaborators have carried out large molecular dynamics simulations to explore a possible magnetic field decay in the inner crust due to the existence of a nuclear pasta phase [74]. In particular, it was concluded that the formation of topological defects in the nuclear pasta could reduce both its electrical and thermal conductivity. Hence, the formation of this "highly resistive layer" could promote magnetic field decay and may ultimately explain the lack of x-ray pulsars with long spin periods [73.

\section{The Outer Core}

Structurally, the stellar core is by far the most critical component of the star. Because of the enormous interior densities, practically all the mass and most of the size reside in the stellar core. At densities of about $10^{14} \mathrm{~g} / \mathrm{cm}^{3}$, the pasta phase "melts" and uniformity in the system is restored. It is in the stellar core where the original perception of Baade and Zwicky [5] is finally realized, namely, a neutron star as a uniform assembly of extremely closed packed neutrons. However, in order to maintain both chemical equilibrium and charge neutrality, a small fraction (of about $10 \%$ ) of protons and leptons is also required. Remarkably, given that the densities in the stellar core are so large, the leptonic component consists of both electrons and muons; indeed, a neutron star typically contains about $10^{56}$ muons! Although exotic, the presence of muons is a model-independent consequence of chemical equilibrium. Instead, the physics of some of the more exotic states of matter that have been speculated to exist in the stellar core - such as hyperons, meson condensates, and quark matter - are much more uncertain. Thus, we limit ourselves to model 
the stellar core exclusively in terms of non-exotic constituents, namely, neutrons, protons, electrons, and muons. Moreover, this approach enables us to test and improve relativistic density functionals that were calibrated using only the properties of finite nuclei. This is highly desirable as the equation of state of dense neutron-rich matter is poorly constrained by laboratory observables.

The cleanest constraint on the EOS at high density emerges from the accurate measurement of massive neutron stars. In this regard, enormous progress has been made by the recent observations of two massive neutron stars by Demorest [13] and Antoniadis [14] at the Green Bank Telescope. In particular, the accurate mass measurement of PSR J1614-2230 (with a mass of $M_{\star}=1.97 \pm 0.04 M_{\odot}$ ) was carried out using "Shapiro delay", a beautiful and powerful technique that relies on the tiny delay in the arrival of the pulsar signal as it "deeps down" into the gravitational well of its white-dwarf companion [13. This observation provides a clear example of the synergy between nuclear physics, astrophysics, and general relativity.

The impact of such mass measurements on the predictions of the mass-vs-radius relation of neutron stars is displayed in Fig. $7 \mathrm{a}$. Note that the constraint from PSR J1614-2230 alone can rule out models with equations of state that are too soft to support a $2 M_{\odot}$ neutron star, such as those that predict the existence of exotic cores. Undoubtedly, the quest for even more massive neutron stars will continue and this will provide unique and valuable insights into the behavior of ultra-dense matter.
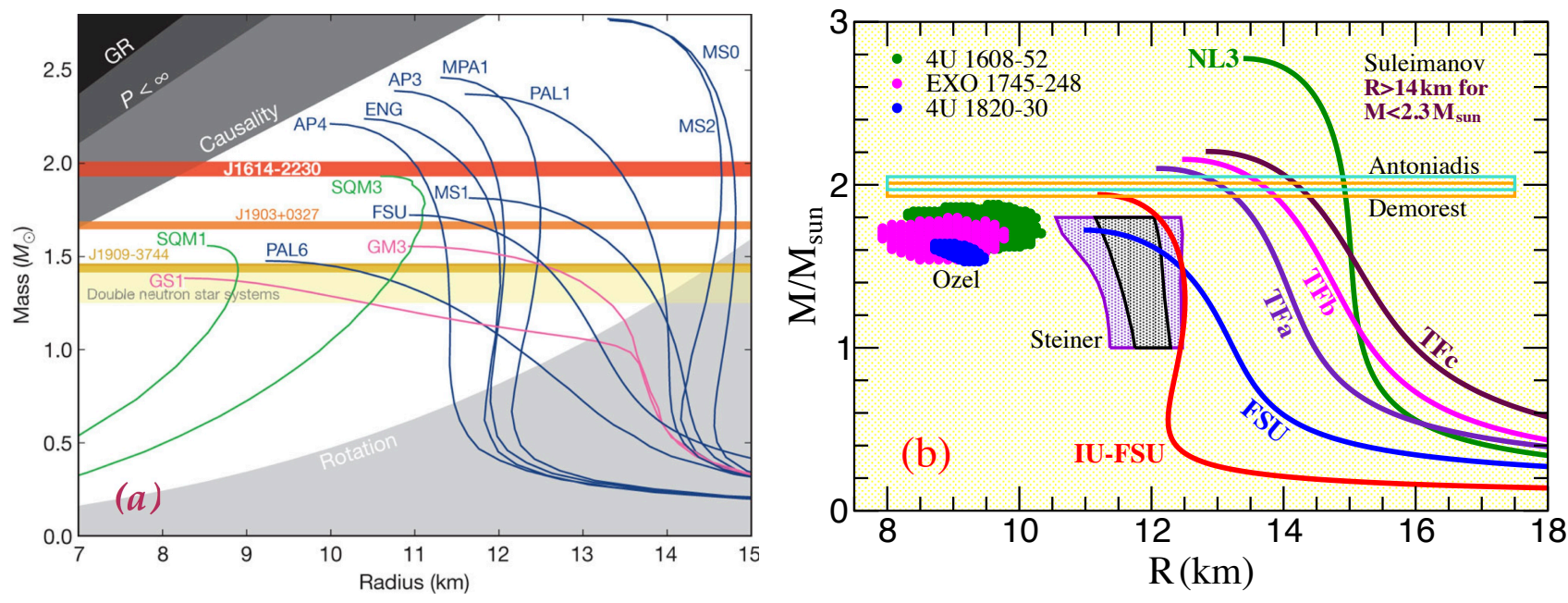

FIG. 7. (Left panel) Predictions for the mass-vs-radius relation for a variety of models of the EOS using both exotic and non-exotic cores. The recent discovery of the massive neutron star PSR J1614-2230 by Demorest and collaborators is clearly indicated in the figure [13. (b) (Right panel) Constraints on both stellar masses and radii extracted from various analyses of x-ray bursts 75 77. Also shown are constraints obtained from the measurement of two massive neutron stars by Demorest 13 and Antoniadis 14 .

Although enormously valuable, the accurate measurement of massive neutron stars does not impose any significant constraint on stellar radii. Indeed, Fig. 7 A indicates that predictions for the radius of a "canonical" $1.4 M_{\odot}$ neutron star can vary by almost a factor of two; between $8 \mathrm{~km}$ and $15 \mathrm{~km}$. Whereas observations of various spectroscopic phenomena in x-ray bursters are promising in the quest to measure stellar radii, the approach currently suffers from large systematic uncertainties. For example, in one of the first analyses of this kind by Özel, Baym, and Güver 75 , it was suggested that neutron stars with masses of about $1.4 M_{\odot}$ have very small radii of about $8-10 \mathrm{~km}$; see Fig. $7 \mathrm{~b}$. This conclusion seems to favor models with exotic cores 78 which, in turn, are disfavored by the observation of $2 M_{\odot}$ neutron stars. However, it was recognized soon after, first by Steiner, Lattimer, and Brown [76] and shortly after by Suleimanov [77, that if one corrects for systematic uncertainties in the analysis by Özel, then it is possible to obtain larger (in the case of the former) or even much larger (in the case of the latter) stellar radii. Indeed, the results by Steiner [76] depicted in Fig.7p by the two shaded areas that indicate $1 \sigma$ and $2 \sigma$ contours, suggest stellar radii in the 10-13 km range. However, even this more conservative limit has been challenged by Suleimanov, who has proposed a lower limit on the stellar radius of $14 \mathrm{~km}$ for neutron stars with masses below $2.3 \mathrm{M}_{\odot}$ [77. This conclusion suggests that the neutron-star matter EOS must be stiff, in agreement with the recent measurement of massive stars.

While at present a reliable technique based on thermal emissions from x-ray bursts has not yet been realized, a recent analysis limited to the observation of accreting neutron stars during quiescence, the so-called quiescent low-mass $x$-ray binaries (qLMXBs), has challenged our current understanding of the equation of state of dense matter. qLMXBs are particularly attractive as they appear to be free from some of the uncertainties that plague x-ray bursters. By analyzing 
the thermal spectra of five qLMXBs inside globular clusters, Guillot and collaborators have reported a common radius for all five sources of $R_{N S}=9.1_{-1.5}^{+1.3} \mathrm{~km}$ at a $90 \%$ confidence level [79]. One should mention that whereas the approach developed in Ref. [79] provides a careful accounting of all uncertainties, some of the assumptions - such as the single common radius - and some of the adopted uncertainties have been called into question [80. Regardless, it is interesting to note that few, if any, nuclear density functionals - whereas relativistic or non-relativistic - can accommodate both a small stellar radius and a large limiting mass [79]. However, we are confident that with new space missions - such as GAIA - that will provide unprecedented positional measurements, many of the current problems plaguing such challenging analyses will be mitigated.

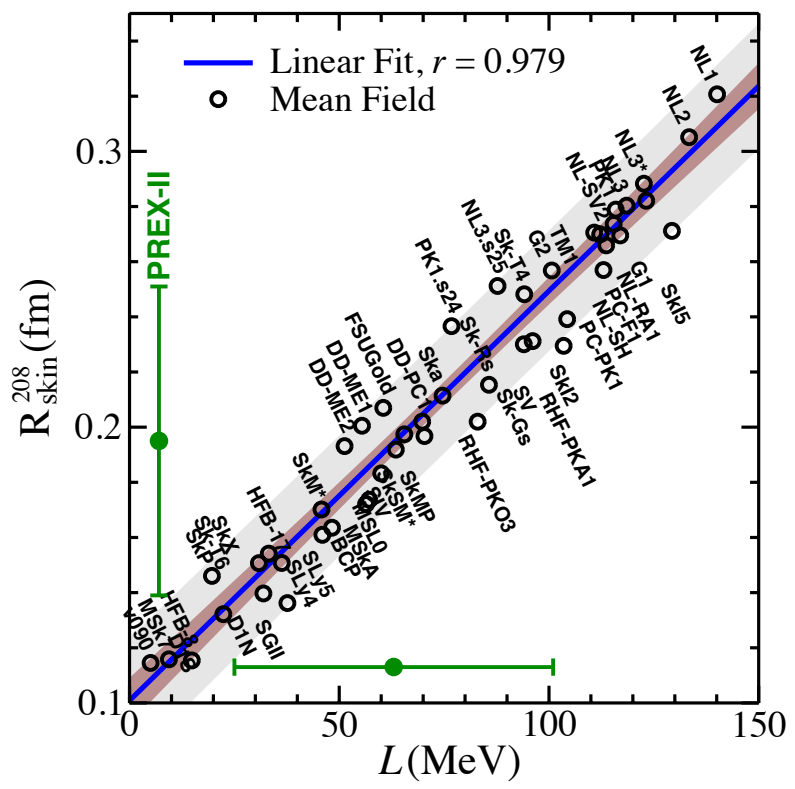

FIG. 8. Predictions from a large number of nuclear density functionals for the neutron-skin thickness of ${ }^{208} \mathrm{~Pb}$ and the slope of the symmetry energy $L$ [81. Constraints from an anticipated upgraded PREX measurement ("PREX-II") have been incorporated into the plot.

Whereas laboratory experiments are of marginal utility in constraining the limiting mass of a neutron star, they play an essential role in constraining stellar radii. This is because the radius of a neutron star is sensitive to the density dependence of the symmetry energy in the immediate vicinity of nuclear-matter saturation density 82 . A fundamental property of the EOS that has received considerable attention over the last decade is the slope of the symmetry energy at saturation density [39. As already shown in Eq. (5), the slope of the symmetry energy $L$ is proportional to the pressure of pure neutron matter at saturation density. In turn, the slope of the symmetry energy is also strongly correlated to a myriad of neutron-star observables [28, 35, 83, 84. Remarkably, $L$ is also strongly correlated to the thickness of the neutron skin of heavy nuclei 85, 86, which is defined as the difference between the neutron and proton root-mean-square radii. The physical reason behind this correlation is particularly insightful. Heavy nuclei favor a neutron excess as a result of the repulsive Coulomb interaction between the protons. Energetically, it is advantageous - to both the surface tension and to the symmetry energy - to form an isospin symmetric $(N=Z)$ core. So the basic question to be answered is where do the extra neutrons go? Placing them in the core reduces the surface tension but increases the symmetry energy. Moving the excess neutrons to the surfaces increases the surface tension but reduces the symmetry energy, which is lower in the dilute surface than in the dense core. So the thickness of the neutron skin emerges from a competition between the surface tension and the difference between the value of the symmetry energy at the surface relative to that at the center; this difference is encoded in the slope of the symmetry energy $L$ [87. Hence, if $L$ is large, then it is energetically advantegeous to move the neutrons to the surface, which results in a thick neutron skin. This suggests a powerful correlation: the larger the value of $L$ the thicker the neutron skin [35. The strong correlation between $L$ and the neutron-skin thickness of ${ }^{208} \mathrm{~Pb}\left(R_{\text {skin }}^{208}\right)$ has been verified using a large and representative set of density functionals - both relativistic and non-relativistic - and is displayed in Fig. 88 [81]. The strong correlation coefficient of $r=0.979$ suggests how a laboratory observable such as $R_{\text {skin }}^{208}$ may serve to determine a fundamental parameter of the equation of state.

Recently, the Lead Radius Experiment ("PREX") at the Jefferson Laboratory has provided the first modelindependent evidence in favor of a neutron-rich skin in ${ }^{208} \mathrm{~Pb}$ 88, 89. Given that the $Z^{0}$ boson couples strongly 


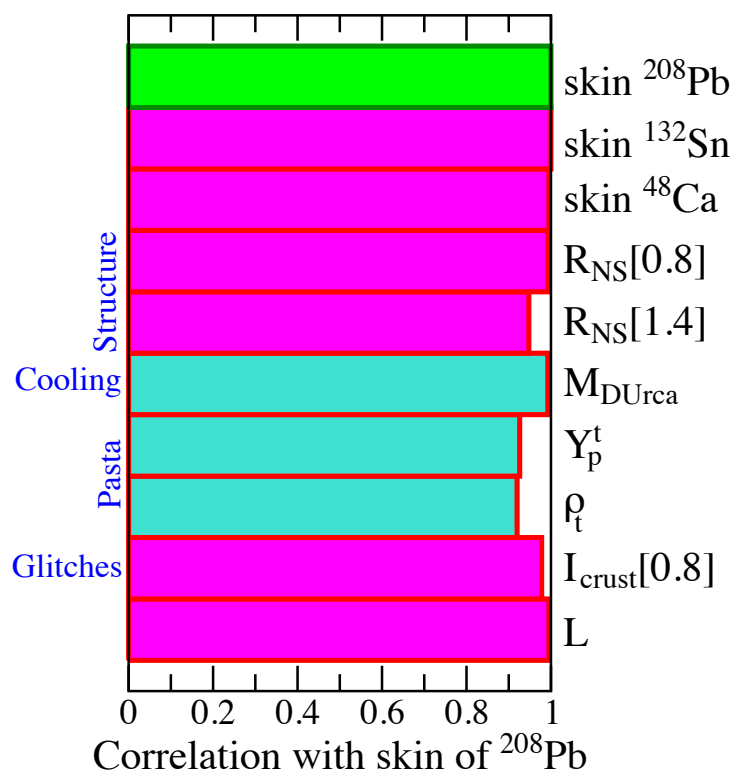

FIG. 9. Correlation coefficients between $R_{\text {skin }}^{208}$ and a variety of neutron-star observables as predicted by the FSUGold density functional [4].

to the neutron, parity violating electron scattering provides a clean probe of neutron densities that is free from strong-interaction uncertainties. As the proton radius of ${ }^{208} \mathrm{~Pb}$ is known extremely accurately from conventional (parity conserving) electron scattering, PREX effectively determined the neutron-skin thickness of ${ }^{208} \mathrm{~Pb}$ to be: $R_{\text {skin }}^{208}=0.33_{-0.18}^{+0.16} \mathrm{fm}[88]$. In a much anticipated and already approved follow-up experiment ("PREX-II") the neutronskin thickness of ${ }^{208} \mathrm{~Pb}$ will be measured with a significantly increased accuracy. The impact of such a measurement in constraining the value of $L$ is displayed with the green error bars in Fig.8.

Perhaps surprisingly, the same pressure that is responsible for creating a neutron-rich skin in heavy nuclei is also responsible for supporting a neutron star against gravitational collapse. Thus, models that predict thicker neutron skins often produce neutron stars with larger radii [28, 35. This makes possible to establish a powerful "data-to-data" relation between the neutron-rich skin of a heavy nucleus and the radius of a neutron star; a relation involving physical observables that differ by 18 orders of magnitude! Such powerful connection is nicely illustrated in Fig. 9 which displays a strong correlation between $R_{\text {skin }}^{208}$ and the stellar radius of a $0.8 M_{\odot}$ neutron star. In the case of a $1.4 M_{\odot}$ neutron star, the correlation weakens slightly because, unlike low-mass neutron stars, medium-to-heavy neutron stars are also sensitive to the symmetry energies at densities above saturation density [36. Moreover, Fig. 9 also displays the enormous reach that an accurate measurement of $R_{\text {skin }}^{208}$ could have on several neutron-star properties [84; note that red bands imply a direct correlation whereas the blue bands an inverse correlation (i.e., an anticorrelation). Indeed, an accurate measurement of $R_{\text {skin }}^{208}$ could have a strong impact on a variety of neutron-star observables related to their structure, cooling, and glitch mechanism [90. We underscore that all properties displayed in Fig.9 - whereas pertaining to finite nuclei or neutron stars - were computed with a unique relativistic energy density functional.

\section{SUMMARY AND OUTLOOK}

Neutron stars are truly gold mines for the study of physical phenomena across a variety of disciplines ranging from elementary particle physics to general relativity. Indeed, binary pulsars have been used to conduct some of the most stringent tests of general relativity and their merger may provide both a powerful source of gravitational waves as well as a plausible site for r-process nucleosynthesis. Moreover, some of the fascinating phases predicted to exist in the neutron-star crust, such as Coulomb crystals of neutron-rich nuclei and nuclear pasta, have counterparts in condensed-matter physics. Finally, the stellar core may harbor new states of matter that may consist of deconfined quark matter, such as color superconductors.

Of course, from the perspective of nuclear physics, neutron stars hold the answer to one of the most fundamental questions in the field: How does subatomic matter organize itself and what phenomena emerge? Indeed, this question figures prominently in the recent community report entitled "Nuclear Physics: Exploring the Heart of Matter". In 
this contribution we challenged the original view of Baade and Zwicky that neutron stars consist of extremely closed packed neutrons [5]. Although undoubtedly such is the most common perception of a neutron star, we showed that the reality is far different and much more interesting. In particular, during our journey through a neutron star we uncovered a myriad of exotic states of matter that are speculated to exist in a neutron star. Moreover, we underscored the critical role that laboratory experiments will play in elucidating the fascinating nature of these exotic states. By the same token, we highlighted the fundamental role that observation of neutron stars using telescopes operating over an enormous range of frequencies will have in constraining the equation of state of neutron-rich matter. As such, neutron stars provide a powerful intellectual bridge between Nuclear Physics and Astrophysics.

Whereas the main goal of the present contribution was an introduction to the fascinating world of neutron stars, the powerful tool used for their study, namely, relativistic density functional theory represents the overarching theme of this whole volume. Density functional theory provides a powerful - and perhaps unique - framework for the accurate calculation of nuclear properties. Based on the seminal work by Kohn and collaborators, DFT shifts the focus from the complicated many-body wave function to the much simpler one-body density. By doing so, the formidable challenge of deducing the exact ground-state energy and one-body density from the many-body wave function "reduces" to the minimization of a suitable functional of the density. Whereas the implementation of DFT to the understanding of electronic properties of materials (work for which Walter Kohn was recognized in 1998 with the Nobel prize in chemistry) is firmly based on the Coulomb interaction, the situation is significantly more difficult in the nuclear physics domain. This is because the parameters of the nuclear density functional can not be computed directly from QCD. By necessity then, the parameters of the model must be directly calibrated (i.e., fitted) to physical observables. However, by directly fitting to data, the short-distance structure of the theory as well as many other complicated many-body effects get implicitly encoded in the parameters of the model. In this regard, DFT provides a unified and powerful approach that may be used to compute physical phenomena ranging over many distance scales, such as the properties of finite nuclei and neutron stars; systems that differ in size by 18 orders of magnitude! Moreover, by implementing the calibration of the model parameters via a standard optimization procedure, DFT becomes a powerful microscopic theory that both predicts and provides well-quantified theoretical uncertainties [18. Documenting theoretical uncertainties is particularly critical as one extrapolates into uncharted regions of the observable landscape.

In this contribution we relied on relativistic DFT to explore three regions of the neutron star: the outer and inner crust as well as the outer core. We determined that the outer stellar crust consists of a Coulomb crystal of neutronrich nuclei embedded in a uniform electron gas. Remarkably, the dynamics of the outer crust is only sensitive to the masses of both stable and unstable nuclei. Thus, mass measurements of exotic nuclei at rare isotope facilities will be instrumental in both constraining theoretical models as well as guiding the extrapolations into regions of the nuclear chart where mass measurement are unlikely to occur. Moving into the inner crust, we established that at the top layers the Coulomb crystal of neutron-rich nuclei is now in equilibrium with a dilute, and likely superfluid, neutron vapor. Hence, in this region one is sensitive to the equation of state of dilute neutron matter, a topic of enormous interest and one that holds an intimate connection to cold atomic Fermi gases in the unitary regime. Given that the EOS of neutron-star matter must span an enormous range of densities, a study of dilute neutron matter can be used to further constrain the form of the relativistic density functional. As one moves even deeper into the inner crust, one discovers a complex pasta phase that displays unique and fascinating dynamical features. Although finding clear signatures of its existence has proved elusive, the lack of x-ray pulsars with long spin periods has recently been suggested as the first observable manifestation of the nuclear pasta phase. Recent molecular dynamics simulations seem to support such an assertion.

Finally, we explored the deep stellar core which consists of a uniform quantum liquid of neutrons, protons, electrons and muons in chemical equilibrium. In this region the relativistic character of the density functional is particularly critical, as it provides a Lorentz covariant extrapolation to the high-density regime. That is, the relativistic nature of the functional guarantees that the speed of sound in matter will always remain below the speed of light at all densities, a feature that is often not displayed by non-relativistic density functionals. Whereas one of the main goals of a relativistic density functional is to provide a unified description of phenomena that happens at many distance scales, we particularly highlighted the strong correlation between a fundamental property of finite nuclei-namely, the thickness of the neutron skin - and the radius of a neutron star. Thus, a laboratory measurement of the neutronskin thickness of ${ }^{208} \mathrm{~Pb}(\mathrm{PREX})$ can constrain the radius of a neutron star. Conversely, accurate measurements of stellar radii can help refine the functional. In particular, we discussed how a recent analysis of quiescent low-mass x-ray binaries seem to favor neutron stars with small stellar radii. Such a finding, which seems to favor a soft EOS, poses serious challenges to theoretical models that must simultaneously account for massive neutron stars of at least 2 solar masses. Moreover, small stellar radii also appear at odds with the original PREX report of a fairly large neutron-skin thickness in ${ }^{208} \mathrm{~Pb}$, albeit with large error bars. However, if future laboratory experiments and astronomical observations confirm that both $R_{\text {skin }}^{208}$ is thick and stellar radii are small, this would strongly suggest a softening of the EOS due to the onset of a phase transition. Regardless, the EOS must eventually stiffen again to account for the existence of massive neutron stars. Such extraordinary behavior will confirm the fundamental role 
of neutron stars as unique laboratories for the study of dense nucleonic matter. Independent of the surprises that may lie ahead, relativistic density functional theory provides the only tractable microscopic theory that can describe nuclear phenomena ranging from the physics of finite nuclei to the structure of neutron stars.

\section{Acknowledgments}

This material is based upon work supported by the United States Department of Energy Office of Science, Office of Nuclear Physics under Award Number DE-FD05-92ER40750.

[1] D. D. Clayton, "Principles of stellar evolution and nucleosynthesis," (University of Chicago Press, Chicago, 1983).

[2] C. Iliadis, "Nuclear physics of stars," (Wiley-VCH, Weinheim, 2007).

[3] S. Chandrasekhar, Astrophys. J 74, 81 (1931).

[4] J. Chadwick, Nature 129, 312 (1932)

[5] W. Baade and F. Zwicky, Phys. Rev. 45, 138 (1934).

[6] J. a. Magueijo, "A brilliant darkness: The extraordinary life and mysterious disappearance of ettore majorana," (Perseus Books Group, New York, NY 10016, 2009).

[7] L. D. Landau, Phys. Z. Sowjetunion 1, 285 (1932), translated into Russian: in Landau L D Sobranie Trudov (Collected Works) Vol. 1 (Moscow: Nauka, 1969) p. 86.

[8] D. G. Yakovlev, P. Haensel, G. Baym, and C. J. Pethick, Phys. Usp. 56, 289 (2013), arXiv:1210.0682 [physics.hist-ph].

[9] J. R. Oppenheimer and G. M. Volkoff, Phys. Rev. 55, 374 (1939).

[10] R. C. Tolman, Phys. Rev. 55, 364 (1939).

[11] A. Hewish, S. Bell, J. Pilkington, P. Scott, and R. Collins, Nature 217, 709 (1968),

[12] J. M. Lattimer, Ann. Rev. Nucl. Part. Sci. 62, 485 (2012), arXiv:1305.3510 [nucl-th].

[13] P. Demorest, T. Pennucci, S. Ransom, M. Roberts, and J. Hessels, Nature 467, 1081 (2010), arXiv:1010.5788 [astro-ph.HE],

[14] J. Antoniadis, P. C. Freire, N. Wex, T. M. Tauris, R. S. Lynch, et al., Science 340, 6131 (2013) arXiv:1304.6875 [astroph.HE].

[15] R. A. Hulse and J. H. Taylor, Astrophys. J. 195, L51 (1975).

[16] M. G. Alford, K. Rajagopal, and F. Wilczek, Nucl. Phys. B537, 443 (1999), arXiv:hep-ph/9804403

[17] M. Kortelainen, T. Lesinski, J. More, W. Nazarewicz, J. Sarich, N. Schunck, M. V. Stoitsov, and S. Wild, Phys. Rev. C 82, 024313 (2010).

[18] PRA-Editors, Phys. Rev. A 83, 040001 (2011).

[19] J. D. Walecka, Annals Phys. 83, 491 (1974).

[20] B. Serot, Phys.Lett. B86, 146 (1979)

[21] R. J. Furnstahl and B. D. Serot, Comments Nucl. Part. Phys. 2, A23 (2000), nucl-th/0005072

[22] P. Hohenberg and W. Kohn, Phys.Rev. 136, B864 (1964)

[23] W. Kohn and L. J. Sham, Phys. Rev. 140, A1133 (1965)

[24] W. Kohn, Rev. Mod. Phys. 71, 1253 (1999).

[25] B. D. Serot and J. D. Walecka, Adv. Nucl. Phys. 16, 1 (1986).

[26] H. Mueller and B. D. Serot, Nucl. Phys. A606, 508 (1996), nucl-th/9603037.

[27] B. D. Serot and J. D. Walecka, Int. J. Mod. Phys. E6, 515 (1997), nucl-th/9701058.

[28] C. J. Horowitz and J. Piekarewicz, Phys. Rev. Lett. 86, 5647 (2001), astro-ph/0010227

[29] R. Furnstahl, B. D. Serot, and H.-B. Tang, Nucl.Phys. A615, 441 (1997) arXiv:nucl-th/9608035 [nucl-th],

[30] R. J. Furnstahl, B. D. Serot, and H.-B. Tang, Nucl. Phys. A618, 446 (1997), nucl-th/9611046

[31] J. J. Rusnak and R. Furnstahl, Nucl.Phys. A627, 495 (1997), arXiv:nucl-th/9708040 [nucl-th]

[32] R. Furnstahl and J. C. Hackworth, Phys.Rev. C56, 2875 (1997) arXiv:nucl-th/9708018 [nucl-th].

[33] M. Kortelainen, R. Furnstahl, W. Nazarewicz, and M. Stoitsov, Phys.Rev. C82, 011304 (2010), arXiv:1005.2552 [nucl-th]

[34] J. Boguta and A. R. Bodmer, Nucl. Phys. A292, 413 (1977).

[35] C. J. Horowitz and J. Piekarewicz, Phys. Rev. C64, 062802 (2001), nucl-th/0108036

[36] J. Carriere, C. J. Horowitz, and J. Piekarewicz, Astrophys. J. 593, 463 (2003), nucl-th/0211015

[37] C. J. Horowitz, M. A. Perez-Garcia, and J. Piekarewicz, Phys. Rev. C69, 045804 (2004), astro-ph/0401079.

[38] B. G. Todd and J. Piekarewicz, Phys. Rev. C67, 044317 (2003), nucl-th/0301092.

[39] J. Piekarewicz and M. Centelles, Phys. Rev. C79, 054311 (2009), arXiv:0812.4499 [nucl-th]

[40] N. Chamel and P. Haensel, Living Rev. Rel. 11, 10 (2008), arXiv:0812.3955 [astro-ph]

[41] C. Bertulani and J. Piekarewicz, "Neutron star crust." (Nova Science Publishers, Hauppauge New York, 2012).

[42] D. Page and S. Reddy, Ann. Rev. Nucl. Part. Sci. 56, 327 (2006), arXiv:astro-ph/0608360 [astro-ph].

[43] G. Baym, C. Pethick, and P. Sutherland, Astrophys. J. 170, 299 (1971).

[44] X. Roca-Maza and J. Piekarewicz, Phys. Rev. C78, 025807 (2008), arXiv:0805.2553 [nucl-th]

[45] R. A. Coldwell-Horsfall and A. A. Maradudin, Journal of Mathematical Physics 1, 395 (1960).

[46] C. A. Sholl, Proceedings of the Physical Society 92, 434 (1967).

[47] B. G. Todd-Rutel and J. Piekarewicz, Phys. Rev. Lett 95, 122501 (2005), nucl-th/0504034 
[48] G. A. Lalazissis, J. Konig, and P. Ring, Phys. Rev. C55, 540 (1997), nucl-th/9607039

[49] G. A. Lalazissis, S. Raman, and P. Ring, At. Data Nucl. Data Tables 71, 1 (1999).

[50] P. Möller, J. R. Nix, W. D. Myers, and W. J. Swiatecki, Atom. Data Nucl. Data Tabl. 59, 185 (1995), arXiv:nuclth/9308022

[51] P. Möller, J. R. Nix, and K. L. Kratz, Atom. Data Nucl. Data Tabl. 66, 131 (1996).

[52] J. Duflo, Nucl. Phys. A576, 29 (1994).

[53] A. Zuker, Nucl. Phys. A576, 65 (1994)

[54] J. Duflo and A. Zuker, Phys. Rev. C 52, R23 (1995)

[55] M. Wang, G. Audi, A. Wapstra, F. Kondev, M. MacCormick, X. Xu, and B. Pfeiffer, Chinese Phys. C 36, 1603 (2012)

[56] S. Goriely, N. Chamel, and J. Pearson, Phys.Rev. C82, 035804 (2010), arXiv:1009.3840 [nucl-th]

[57] R. Wolf et al. (ISOLTRAP Collaboration), Phys. Rev. Lett. 110, 041101 (2013).

[58] D. G. Ravenhall, C. J. Pethick, and J. R. Wilson, Phys. Rev. Lett. 50, 2066 (1983).

[59] M. Hashimoto, H. Seki, and M. Yamada, Prog. Theor. Phys. 71, 320 (1984).

[60] A. L. Fetter and J. D. Walecka, "Quantum theory of many particle systems," (McGraw-Hill, New York, 1971).

[61] R. Jamei, S. Kivelson, and B. Spivak, Phys. Rev. Lett. 94, 056805 (2005).

[62] C. J. Horowitz, M. A. Perez-Garcia, J. Carriere, D. K. Berry, and J. Piekarewicz, Phys. Rev. C70, 065806 (2004), astro-ph/0409296.

[63] C. J. Horowitz, M. A. Perez-Garcia, D. K. Berry, and J. Piekarewicz, Phys. Rev. C72, 035801 (2005), nucl-th/0508044

[64] G. Watanabe, K. Sato, K. Yasuoka, and T. Ebisuzaki, Phys. Rev. C68, 035806 (2003), arXiv:nucl-th/0308007 [nucl-th]

[65] G. Watanabe, T. Maruyama, K. Sato, K. Yasuoka, and T. Ebisuzaki, Phys. Rev. Lett. 94, 031101 (2005), arXiv:nuclth/0408061 [nucl-th]

[66] G. Watanabe, H. Sonoda, T. Maruyama, K. Sato, K. Yasuoka, et al., Phys. Rev. Lett. 103, 121101 (2009), arXiv:0904.0512 [nucl-th]

[67] T. Maruyama, T. Tatsumi, D. N. Voskresensky, T. Tanigawa, and S. Chiba, Phys. Rev. C72, 015802 (2005), arXiv:nuclth/0503027 [nucl-th]

[68] S. Avancini, D. Menezes, M. Alloy, J. Marinelli, M. Moraes, et al., Phys. Rev. C78, 015802 (2008).

[69] S. Avancini, L. Brito, J. Marinelli, D. Menezes, M. de Moraes, et al., Phys.Rev. C79, 035804 (2009), arXiv:0812.3170 $[$ nucl-th]

[70] W. Newton and J. Stone, Phys. Rev. C79, 055801 (2009).

[71] G. Shen, C. Horowitz, and S. Teige, Phys. Rev. C83, 035802 (2011), arXiv:1101.3715 [astro-ph.SR],

[72] J. Piekarewicz and G. Toledo Sanchez, Phys.Rev. C85, 015807 (2012) arXiv:1107.2638 [nucl-th].

[73] J. A. Pons, D. Vigano; and N. Rea, Nature Physics, 9, 431-434 (2013), 10.1038/nphys2640, arXiv:1304.6546 [astro-ph.SR]

[74] C. Horowitz, D. Berry, C. Briggs, M. Caplan, A. Cumming, et al., (2014), arXiv:1410.2197 [astro-ph.HE].

[75] F. Ozel, G. Baym, and T. Guver, Phys. Rev. D82, 101301 (2010), arXiv:1002.3153 [astro-ph.HE]

[76] A. W. Steiner, J. M. Lattimer, and E. F. Brown, Astrophys. J. 722, 33 (2010), arXiv:1005.0811 [astro-ph.HE].

[77] V. Suleimanov, J. Poutanen, M. Revnivtsev, and K. Werner, Astrophys. J. 742, 122 (2011), arXiv:1004.4871 [astro-ph.HE]

[78] F. J. Fattoyev and J. Piekarewicz, Phys. Rev. C82, 025805 (2010), arXiv:1003.1298 [nucl-th].

[79] S. Guillot, M. Servillat, N. A. Webb, and R. E. Rutledge, Astrophys. J. 772, 7 (2013), arXiv:1302.0023 [astro-ph.HE]

[80] J. M. Lattimer and A. W. Steiner, (2013), arXiv:1305.3242 [astro-ph.HE]

[81] X. Roca-Maza, M. Centelles, X. Vinas, and M. Warda, Phys. Rev. Lett. 106, 252501 (2011), arXiv:1103.1762 [nucl-th]

[82] J. M. Lattimer and M. Prakash, Phys. Rept. 442, 109 (2007), arXiv:astro-ph/0612440

[83] C. J. Horowitz and J. Piekarewicz, Phys. Rev. C66, 055803 (2002), nucl-th/0207067

[84] F. Fattoyev and J. Piekarewicz, Phys. Rev. C88, 015802 (2012), arXiv:1203.4006 [nucl-th]

[85] B. A. Brown, Phys. Rev. Lett. 85, 5296 (2000).

[86] R. J. Furnstahl, Nucl. Phys. A706, 85 (2002), nucl-th/0112085

[87] C. Horowitz, E. Brown, Y. Kim, W. Lynch, R. Michaels, et al., J. Phys. G41, 093001 (2014), arXiv:1401.5839 [nucl-th].

[88] S. Abrahamyan, Z. Ahmed, H. Albataineh, K. Aniol, D. Armstrong, et al., Phys.Rev.Lett. 108, 112502 (2012) arXiv:1201.2568 [nucl-ex].

[89] C. Horowitz, Z. Ahmed, C. Jen, A. Rakhman, P. Souder, et al., Phys.Rev. C85, 032501 (2012), arXiv:1202.1468 [nucl-ex]

[90] F. J. Fattoyev and J. Piekarewicz, Phys. Rev. C82, 025810 (2010), arXiv:1006.3758 [nucl-th]. 